Medizinisch-ethische Richtlinien der SAMW zur Behandlung und Betreuung von zerebral schwerstgeschädigten Langzeitpatienten Antwort auf den zweimal publizierten Leserbrief von Dr. med. A. Oberle [1, 2]

Implizit ergeht hier ein Aufruf, Wege zu finden, ein als «sinnlose Last» und «nicht mehr menschlich empfundenes Leben (nach gewissenhafter Beurteilung)» abzubrechen. Konkret hiesse das, die aktive ärztliche Sterbehilfe für den Moment einzuführen, wo jeweils die Entscheidung getroffen wird, die verbleibenden Restchancen auf eine neurologische Verbesserung $\mathrm{zu}$ negieren und den vegetativen Status als «permanent» $\mathrm{zu}$ bezeichnen.

Jedem Individuum steht es frei, sein eigenes Leben ab einem bestimmten Punkt der Hilfsbedürftigkeit und Behinderung als nicht weiter lebenswert einzuschätzen. Wenn aber die eigene Betroffenheit dazu verleitet, die ethischen, prognostischen und erkenntnis-theoretischen Dilemmata beim Wachkoma so weit zu vereinfachen, dass schon die Würde des Menschen im vegetativen Status in Abrede gestellt wird («nur noch ein immobiler Corpus mit rudimentären Funktionen»), und dies als abfärbend und gefährdend gleich mit «für die Würde der ihn Pflegenden» gesehen wird, so liegt eine Grenzüberschreitung vor, die wir ablehnen.

Die hier vorgebrachte utilitaristische Sichtweise von Pflegetätigkeit bedroht langfristig Sinn und Wert der gesellschaftlichen Zuwendung nicht nur für zerebral schwerstgeschädigte Langzeitpatienten, sondern für einen von Gesunden beliebig zu definierenden Randbereich der Gesellschaft, wozu als erstes vermutlich auch die aus dem vegetativen Status «Erwachten» gehören könnten, die im sogenannten «minimal responsive state» weiterhin hoher Hilfestellung bedürfen. Sie bedroht auch eine von Angehörigen und Pflegenden oft geäusserte und gelebte Haltung. Es ist die nicht selten prekäre pflegerische Un- terversorgung, welche die (unveräusserliche) Würde des Menschen im Wachkoma im Sinne einer Entwürdigung aufzeigt. Die Schwierigkeiten bei der Suche nach einem Pflegeplatz und der latent zu spürende Widerstand sind nach unserer Erfahrung eben nicht die Folge einer fehlenden pflegerischen Motivation, sondern einer falschen Bedarfsplanung und unzureichender Ressourcen. Dies belastet die Angehörigen oft ebensostark wie der Schicksalsschlag der Erkrankung selbst.

Forschungsaufgaben zum Thema Wachkoma werden nicht nur wegen der schwierigen methodischen Voraussetzungen wenig bearbeitet, sondern auch wegen minimaler Mittel, die dafür bereitstehen. «Ignorabimus» wird weiter über den ungelösten Fragen zum Langzeitverlauf und zum Charakter des Bewusstseins bei schwerster Hirnschädigung stehen, wenn die Akzeptanz dafür wächst, sich dieser menschlichen Lebensform $\mathrm{zu}$ angemessener Zeit (aus humanitären Gründen) zu entledigen. Hier ist eine Wohlstandsgesellschaft trotz aller Sparzwänge gefordert, vor der Kehrseite von erfolgreicher Spitzenmedizin bei Reanimationen und Neurochirurgie nicht die Augen zu verschliessen und das abwartende Offenlassen der unsicheren Prognose für Patienten im vegetativen Status zuzulassen - für die Dauer der fachspezifischen Rehabilitation und für die pflegerischen Bedürfnisse danach.

Dr. med. Peer Schleyerbach, Dr. med. Christian Kätterer, Sabine Schmid, Dr. med. Mark Mäder REHAB Basel, Zentrum für Querschnittsgelähmte und Hirnverletzte

1 Oberle A. Medizinisch-ethische Richtlinien der SAMW zur Behandlung und Betreuung von zerebral schwerstgeschädigten Langzeitpatienten. Schweiz Ärztezeitung 2004;85(8):377.

2 Oberle A. Medizinisch-ethische Richtlinien der SAMW zur Behandlung und Betreuung von zerebral schwerstgeschädigten Langzeitpatienten. Schweiz Ärztezeitung 2004;85(10):498. 


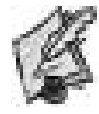

\section{Elektronische Abrechnung}

TARMED-Info Bulletin Nr. 19 [1] klärt uns lapidar auf: «Solange die Versicherer nicht in der Lage sind, die Rechnungen elektronisch zu verarbeiten, werden sie als nicht elektronisch übermittelt erachtet, und es kommen die diesbezüglichen Limitationen im TARMED zur Anwendung.» Limitationen, welche fast ausschliesslich Grundversorger treffen, denen ja eine Verbesserung ihrer finanziellen Abgeltung versprochen wurde. Die Versicherer wären ja dumm, uns elektronisch abrechnen zu lassen.

Vor Tische las es sich anders: Im Dezember bestätigte mir der Tarifdienst FMH schriftlich, dass die Teilnahme an einem Trustcenter das Kriterium der elektronischen Abrechnung erfülle und somit die unsäglichen Limitationen entfallen. Eine gleichlautende Anfrage beim kantonalen Krankenkassenverband santésuisse Fribourg wurde während eines Monates überhaupt nicht und nach Nachstossen sehr kompetent beantwortet: Ich soll mich mit dieser Frage an meinen Berufsverband wenden (!).

O.K. - um den Limitationen zu entgehen, unterschrieb ich einen Vertrag mit Ctésias, dem Trustcenter der Romandie, weil das bernische Trustcenter uns Deutschfreiburger nicht aufnehmen durfte (wieso nicht?). Die kürzestmögliche Vertragsdauer bei Ctésias beträgt zwei Jahre - ergibt Abonnementskosten von Fr. 1360.-. Auch die Lizenz für die Schnittstelle (Fr. 620.-) wurde freudig gelöst.

Und nun? Die Versicherer senden mir meine Rechnungen (tiers garant) nach langer Bearbeitungsdauer zurück mit der Aufforderung, ihren Versicherten die zu Unrecht verrechneten $>3$ Pos. 00.0020 zurückzuerstatten.

Und ich fordere die betroffenen Patienten auf, sich Gedanken über den Zusammenhang zwischen der Seriosität meiner ärztlichen Bemühungen und einer elektronischen Abrechnung zu machen - und die Krankenkasse zu verlassen, die diesen absurden Link durchsetzen will, indem sie von der Möglichkeit dieser Abrechnungsart nicht Gebrauch macht. Und natürlich turne ich von nun an ausserhalb 00.0020 durch meinen Zeitaufwand.

Dr. med. G. Baumgartner, Murten

\section{Persönliche Erklärung}

Am 9. Februar 2003 hatten die Stimmberechtigten im Rahmen einer eidgenössischen Referendumsabstimmung zu einem neuen (und inzwischen in Kraft getretenen) Bundesgesetz über die Anpassung der kantonalen Beiträge für die innerkantonalen stationären Behandlungen nach KVG Stellung zu nehmen. Im Vorfeld dieser Volksabstimmung habe ich einen Artikel verfasst, der in der Schweizerischen Ärztezeitung Nr. 3/2003 erschienen ist [1]. In diesem Artikel wurde unter anderem gesagt, dass die Assura S.A., welche das erwähnte Referendum ergriffen hatte, erst einmal die Gelder bzw. die durch subventionierte Spitalrechnungen gesunkenen Kosten im Zusatzversicherungsbereich an die Versicherten weitergeben sollte, bevor sie nach mehr Geld ruft.

Die Assura S.A. beanstandet an dieser Äusserung, dass daraus der nicht berechtigte Vorwurf hervorgehen könnte, die Assura S.A. habe sich unrechtmässig verhalten, indem sie sich zum Nachteil ihrer Versicherten öffentliche Gelder angeeignet habe. Eine solche Interpretation meines Textes wäre falsch. Einen solchen Vorwurf zu äussern war nicht meine Absicht, und ich bedaure es, wenn mein oben zitierter Text in diesem Sinne verstanden wurde.

\section{Alice Scherrer-Baumann, Landammann des Kantons Appenzell Ausserrhoden}

1 Scherrer-Baumann A. JA zu einer gesunden Spitalfinanzierung am 9. Februar 2003. Schweiz Ärztezeitung 2003;84(3):71-2.

1 Büro TARMED. TARMED-Info. Bulletin Nr. 19. Schweiz Ärztezeitung 2004;85(13):661-4. 